\title{
Haemophilus influenzae meningitis 5 years after introduction of the Haemophilus influenzae type b conjugate vaccine in Brazil
}

\author{
Guilherme S. Ribeiro $^{\text {a }}$, Josilene B.T. Lima ${ }^{a}$, Joice N. Reis ${ }^{a}$, , Edilane L. Gouveia $^{\text {a }}$, \\ Soraia M. Cordeiro ${ }^{a}$, Tatiana S. Lobo ${ }^{a}$, Ricardo M. Pinheiro ${ }^{a}$, Cássio T. Ribeiro ${ }^{a}$, \\ Alan B. Neves ${ }^{\text {a }}$, Kátia Salgado ${ }^{c}$, Hagamenon R. Silva ${ }^{\text {c,d }}$, \\ Mitermayer G. Reis ${ }^{a}$, Albert I. Ko ${ }^{\mathrm{a}, \mathrm{e}, *}$

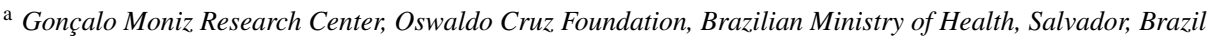 \\ b School of Pharmacy, Federal University of Bahia, Salvador, Brazil \\ ${ }^{\mathrm{c}}$ Hospital Couto Maia, Secretary of Health for the State of Bahia, Salvador, Brazil \\ d School of Medicine, Federal University of Bahia, Salvador, Brazil \\ e Division of International Medicine and Infectious Diseases, Department of Medicine, Weill Medical College of Cornell University, NY, USA
}

Received 15 November 2006; received in revised form 6 March 2007; accepted 13 March 2007

Available online 30 March 2007

\begin{abstract}
The long-term impact of Haemophilus influenzae type b (Hib) conjugate vaccine, introduced throughout Latin America in the late 1990s, has not been evaluated. Active surveillance for $H$. influenzae meningitis was performed from August 9, 1996 to August 8, 2004 in Metropolitan Salvador, Brazil. Five years after the introduction of Hib conjugate vaccine, Hib meningitis incidence decreased from 2.39 to 0.06 cases per 100,000 population (98\%) overall, and from 60.9 to 3.1 cases per 100,000 population $(95 \%)$ in children $<1$ year of age. A transient serotype replacement phenomenon was observed associated with a small increase of meningitis due to two $H$. influenzae type a clonal groups. These findings indicate that Hib immunization campaign has led to the virtual elimination of Hib disease in this region.
\end{abstract}

(C) 2007 Elsevier Ltd. All rights reserved.

Keywords: Haemophilus influenzae; Vaccine effectiveness; Serotype replacement

\section{Introduction}

Introduction of Haemophilus influenzae type b (Hib) polysaccharide-protein conjugate vaccines have dramatically reduced the burden of Hib invasive disease in the last 20 years [1-3]. Clinical trials demonstrated that conjugate vaccines have an efficacy of 90-100\% in preventing Hib invasive disease [4,5]. Furthermore, the vaccine has been highly effective, even in populations where coverage is not complete, due to its ability to reduce nasopharyngeal colonization in immu-

\footnotetext{
* Corresponding author at: Centro de Pesquisas Gonçalo Moniz, Fundação Oswaldo Cruz, Ministério da Saúde, Rua Waldemar Falcão, 121, Salvador, Bahia 40296-710, Brazil. Tel.: +55 713176 2302; fax: +55 7131762281 .

E-mail address: aik2001@med.cornell.edu (A.I. Ko).
}

nized individuals $[6,7]$ and induce herd immunity $[8,9]$. In the United States (US) and most industrialized countries, Hib invasive disease has been virtually eliminated [1-3]. Whereas more than 10,000 cases of Hib invasive disease were reported annually in the US prior to introduction of conjugate vaccines, less than 100 cases are now reported each year [1].

The priority for Hib invasive disease is to extend the benefits afforded by conjugate vaccines to developing countries [2]. Worldwide, in $2003,61 \%$ of children in target age groups for immunization did not receive the conjugate vaccine [10]. Hib diseases remain the cause of an estimated 386,000 deaths yearly [11]. However, major progress has been made in South and Central Americas and the Caribbean where Hib conjugate vaccines were introduced in the late 1990s and are used in all but four countries [12]. Although several studies have 
reported the effectiveness of Hib immunization campaigns [13-21], the long-term impact of these programs has not been evaluated in the region.

In Salvador, a city in Northeast Brazil, the Hib immunization program caused a $69 \%$ decrease in the Hib meningitis rate 1 year after its initiation in 1999 [21]. Moreover, we found that a serotype replacement phenomenon had occurred during the same period which led to a small but significant increase in the $H$. influenzae type a meningitis rate [21]. Population-based evidence for serotype replacement had not been reported in other settings in association with the use of Hib conjugate vaccines and may be a transient phenomenon dependent on the presence of circulating virulent non-type $b$ clones. Here, we report findings from active surveillance in Salvador, Brazil conducted to evaluate the impact of the Hib immunization program 5 years after its initiation and determine whether the serotype replacement phenomenon initially described continued to persist.

\section{Materials and methods}

\subsection{Surveillance}

Active surveillance for $H$. influenzae meningitis was performed during the 8-year period from August 9, 1996 to August 8, 2004 in Metropolitan Salvador (population, 3,543,651 inhabitants [22], which includes Salvador, the third largest city in Brazil, and 29 municipalities within a radius of $75 \mathrm{~km}$. Patients were identified at the state infectious disease hospital where according to the state health secretary procedures, all suspected cases of meningitis for the region are referred for diagnostic procedures, including routine lumbar puncture and cerebrospinal fluid examination, and to implement isolation precaution protocols. A study team of physicians and medical students reviewed laboratory records 5 days a week to identify new culture isolations. A case was defined as a patient who had: (1) a clinical presentation of meningitis, characterized by fever, meningismus, and altered mental status; (2) an abnormal cerebrospinal fluid examination; and (3) positive cerebrospinal fluid or blood culture for $H$. influenzae. Reporting of $H$. influenzae meningitis is mandatory and is performed by the epidemiological surveillance staff at the study hospital. The study team reviewed case reports at the state health secretary and determined that cases identified at the surveillance hospital represent $98 \%$ of the cases notified from Metropolitan Salvador between 1996 and 2004 [23]. Patients were enrolled in the study according to informed consent procedures approved by the Institutional Review Boards of the Oswaldo Cruz Foundation, Brazilian Ministry of Health and the New York-Presbyterian Hospital.

\subsection{Hib immunization program}

Hib conjugate vaccine was introduced as part of routine childhood immunization on August 9, 1999. Children <12 months of age received a three-dose immunization schedule, performed at 2nd, 4th and 6th months of age. H. influenzae type $b$ polyribosylribitol phosphate conjugated to tetanus toxoid protein (PRP-T) was used as the vaccine in all surveillance years except for the 1st year when the Hib polysaccharide conjugated to modified diphtheria toxoid CRM197 protein (HbOC) was used. The Hib conjugate vaccine was administered at the same time as the diphtheria-tetanus-whole cell pertussis (DTP) vaccine. In Metropolitan Salvador, 82, 77, 69, 86 and $82 \%$ of the target population completed a three-dose schedule in 2000, 2001, 2002, 2003 and 2004, respectively [24]. During the 1st and 2nd year of the Hib immunization campaign, children with 12-23 months of age received a single dose of the vaccine. The coverage for the catch up immunization program was 37 and $24 \%$ in this age group in 1999 and 2000, respectively [24].

\subsection{Data collection}

A standardized data entry form was used to extract information on demographic, clinical presentation and outcome characteristics from the patient's medical record. Neurological examinations and assessment of sequelae are routinely performed by physicians for patients with bacterial meningitis during hospitalization. Mothers of the patients were interviewed and immunization cards were reviewed to obtain information on the timing and number of Hib conjugate vaccine doses administered prior to hospitalisation.

\subsection{Laboratory investigation}

H. influenzae was identified based on Gram stain morphology and growth requirement for hemin and nicotinamide adenine dinucleotide. Prior to 1999 , isolates were independently serotyped at two research laboratories in Brazil. The slide agglutination method was used to determine serotype with a complete panel of type a to f-specific antisera (Difco Laboratories) and a saline control. A semi-nested polymerase chain reaction (PCR) method that amplifies serotype-specific and non-specific DNA sequences from the $H$. influenzae capsular loci [25] was used to confirm the serotype status for non-type $\mathrm{b}$ isolates and those with discordant serotype results. Beginning in 1999, isolates were routinely typed with serologic and PCR-based methods. Isolates were defined as non-capsulated if agglutination was not observed with the six type-specific antisera and if capsular loci sequences conserved among serotypes were not amplified in the PCR assay [25]. Pulsed-field gel electrophoresis (PFGE) was performed with SmaI-digested DNA of $H$. influenzae type a strains obtained during surveillance [21]. Identical and closely related PFGE typing patterns were defined according to the criteria of Tenover et al. [26].

\subsection{Statistical analysis}

Epi-Info Version 6.04 software (Centers for Disease Control and Prevention, US) was used for data entry and 
statistical analysis. Incidence was calculated based on the number of cases from Metropolitan Salvador and population counts from the 2000 national census [22]. The incidence for culture-positive Neisseria meningitidis and Streptococcus pneumoniae meningitis was determined to evaluate changes in overall case ascertainment which may have occurred during the 8-year surveillance period. Pre and post-vaccine periods were defined as the 3-year interval between August 9, 1996 and August 8, 1999 and the 5-year interval between August 9, 1999 and August 8, 2004, respectively. The percent of change in the $H$. influenzae incidence and $95 \%$ confidence intervals were calculated for each of the post-vaccine years in comparison with the mean incidence during the pre-vaccine period to evaluate the effectiveness of the Hib immunization program. Cases were defined as eligible to receive the Hib conjugate vaccine if they were born after August 9, 1997. The number of vaccine doses received was determined based on immunizations that were administered more than 1 month prior to hospitalization with $H$. influenzae meningitis. The association between Hib conjugate vaccine use and acquisition of $H$. influenzae type a strains was evaluated by comparing the proportions of prior vaccine use among $H$. influenzae type a and non-type a cases identified during the post-vaccine period. The chi-square test or Fisher's exact test was performed to compare proportions. Differences were defined to be significant if the two-tailed $P$-value was $<0.05$.

\section{Results}

\subsection{Surveillance}

During the 8-year period between August 9, 1996 and August 8, 2004, active surveillance identified $522 \mathrm{H}$. influenzae meningitis cases. Among the identified cases, 356 (68.2\%) were residents of Metropolitan Salvador, a region which has a primarily urban-based population and according to the national census in 2000, had 128,576 and 191,423 children less than 2 years and 2-4 years, respectively, of age. The majority of the $H$. influenzae meningitis cases were children less than 2 (321 cases, $61.5 \%$ ) and 2-4 years (148 cases, $28.4 \%$ ) of age. A higher proportion of cases were males than females (56.9\% versus $43.1 \%$, respectively). Among the 483 (92.5\% of 522) cases for whom an isolate was stored and available for serotyping, 458 (94.8\%), $19(3.9 \%), 3(0.6 \%)$, and $3(0.6 \%)$ had $H$. influenzae type b, type a, type e and non-capsulated isolates, respectively (Fig. 1).

\subsection{Clinical outcome}

Overall case fatality was $15.9 \%$ ( 83 of 522). There was no statistically significant difference between case fatality in the pre-vaccine and post-vaccine period (14.8\% versus $19.5 \%$, respectively, $P>0.05$ ). Among survivors, 112 (25.5\% of 439) had neurological sequelae on hospital discharge. The most frequent types of sequelae encountered were ataxia (50 cases,

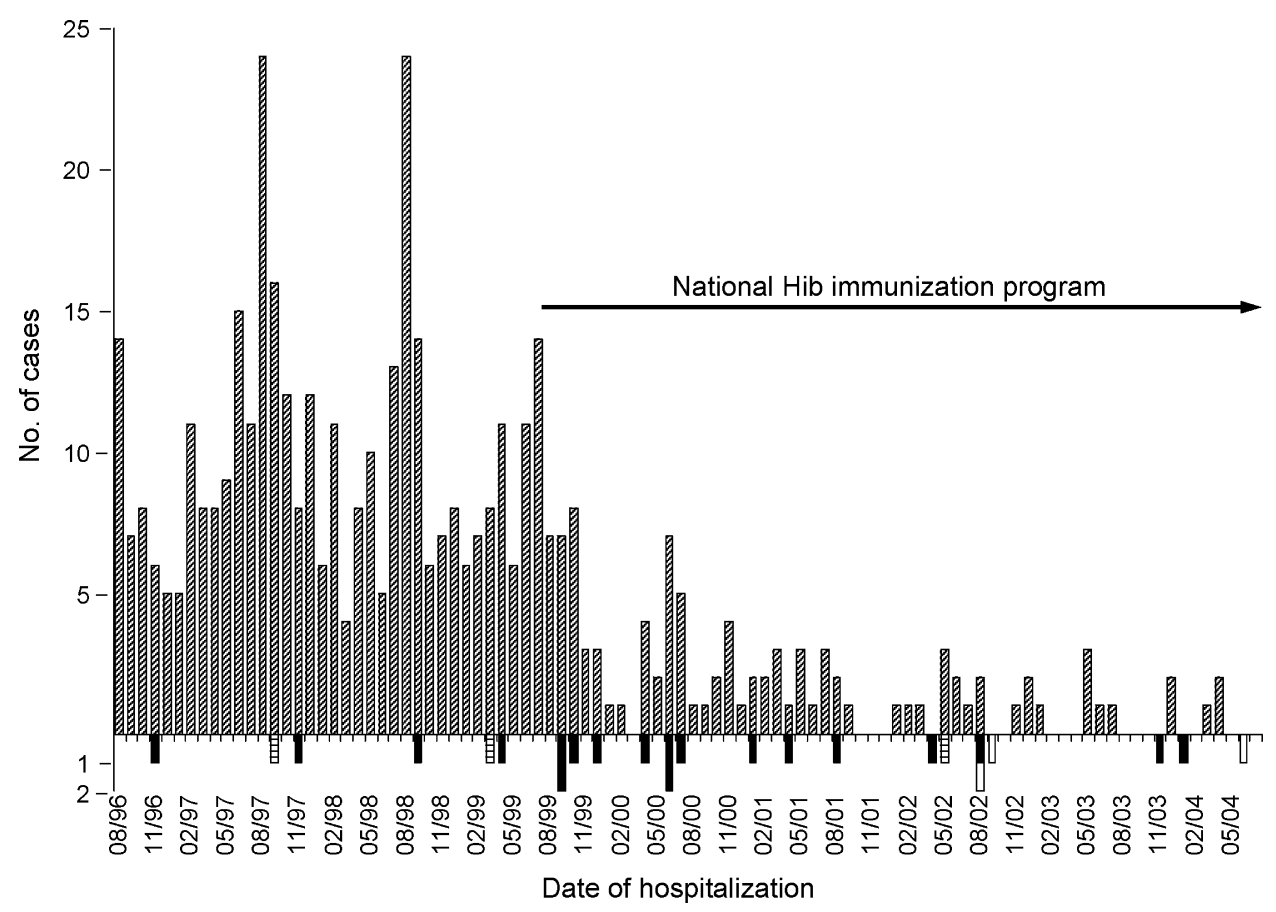

Fig. 1. Distribution of $H$. influenzae meningitis cases $(N=483)$ identified in Salvador, Brazil between August 1996 and July 2004, according to month of hospitalization and isolate serotype. Hib meningitis cases are represented as cross-hatched boxes above the horizontal axis. H. influenzae type a (black box), type e (open box), and non-capsulated (horizontal hatched box) meningitis cases are represented as boxes below the horizontal axis. The Hib immunization campaign was initiated in August 1999. 
Table 1

Incidence of Haemophilus influenzae type b (Hib) meningitis before and after introduction of the Hib immunization program in Salvador, Brazil

\begin{tabular}{|c|c|c|c|c|c|c|c|c|}
\hline \multirow[t]{3}{*}{ Age group } & \multicolumn{8}{|c|}{ Surveillance year $^{\mathrm{a}}$} \\
\hline & \multicolumn{3}{|c|}{ Pre-vaccine period } & \multicolumn{5}{|c|}{ Post-vaccine period } \\
\hline & 1 & 2 & 3 & 4 & 5 & 6 & 7 & 8 \\
\hline & \multicolumn{8}{|c|}{ Cases per 100,000 population (No. of cases ${ }^{b}$ ) } \\
\hline Total & $2.18(77)$ & $2.65(94)$ & $2.34(83)^{\mathrm{c}}$ & $0.85(30)$ & $0.42(15)$ & $0.31(11)$ & $0.14(5)$ & $0.06(2)$ \\
\hline$<5$ years & $23.44(75)$ & $28.44(91)$ & $24.38(78)$ & $8.44(27)$ & $3.44(11)$ & $2.19(7)$ & $1.56(5)$ & $0.63(2)$ \\
\hline$<1$ year & $60.92(39)$ & $62.49(40)$ & $59.36(38)$ & $18.75(12)$ & $7.81(5)$ & $6.25(4)$ & $4.69(3)$ & $3.12(2)$ \\
\hline 1 year & $30.98(20)$ & $43.37(28)$ & $30.98(20)$ & $6.20(4)$ & $1.55(1)$ & $0.00(0)$ & $1.55(1)$ & $0.00(0)$ \\
\hline 2 years & $17.10(11)$ & $21.76(14)$ & $15.54(10)$ & $7.77(5)$ & $1.55(1)$ & $1.55(1)$ & $0.00(0)$ & $0.00(0)$ \\
\hline $3-4$ years & $3.93(5)$ & $7.08(9)$ & $7.87(10)$ & $4.72(6)$ & $3.15(4)$ & $1.57(2)$ & $0.79(1)$ & $0.00(0)$ \\
\hline 5-9 years & $0.63(2)$ & $0.94(3)$ & $1.26(4)$ & $0.94(3)$ & $1.26(4)$ & $1.26(4)$ & $0.00(0)$ & $0.00(0)$ \\
\hline
\end{tabular}

${ }^{a}$ Each surveillance year corresponds to the period between August 9th and August 8th. Surveillance years 1-3 (1996-1999) and 4-8 (1999-2004) are the periods prior to and after, respectively, the introduction of Hib conjugate vaccines in the national childhood immunization program.

b A total of 317 Hib meningitis cases were identified in Metropolitan Salvador during the 8-year surveillance period.

c The total does not match with the sum because there was one patient older than 9 years of age.

$11.4 \%)$, auditory deficit $(21,4.8 \%)$, motor deficit $(20,4.6 \%)$ and hydrocephalus $(19,4.3 \%)$.

\subsection{Population-based analyses}

Of the 483 cases with serotype information, 331 (68.5\%) were residents of Metropolitan Salvador. Hib was the cause of meningitis in 317 (95.8\%) of the 331 cases. Annual incidence of Hib meningitis during the 3-year pre-vaccine period ranged from 2.18 to 2.65 cases per 100,000 population (mean incidence, 2.39 cases per 100,000 population) (Table 1). Children $<1$ year of age were the age group with the highest risk for Hib meningitis (mean incidence, 60.92 cases per 100,000 population) (Table 1 and Fig. 2A). Mean incidences of Hib meningitis among children $<2$ and $<5$ years was 48.01 and
25.42 cases per 100,000 population, respectively, during the pre-vaccine period.

Hib meningitis rates decreased sharply in the $<2$ years age groups in the first years of the Hib immunization campaign (Table 1 and Fig. 2A). In children $<1$ year of age, Hib meningitis incidence decreased by 69.2 and $94.9 \%$, respectively, 1 and 5 years after introduction of the Hib conjugate vaccines (Table 2). Although children 2-4 years of age were not vaccinated during the 1st year (surveillance year 4) of the Hib immunization program, a reduction of 57.1 and $25.0 \%$ was observed in the Hib meningitis incidence for children in the 2 and 3-4 year age groups, respectively (Table 2 and Fig. 2A). All children with $<5$ years of age were eligible to have received Hib immunization by the 5th year of Hib campaign (surveillance year 8 ). Hib meningitis incidence
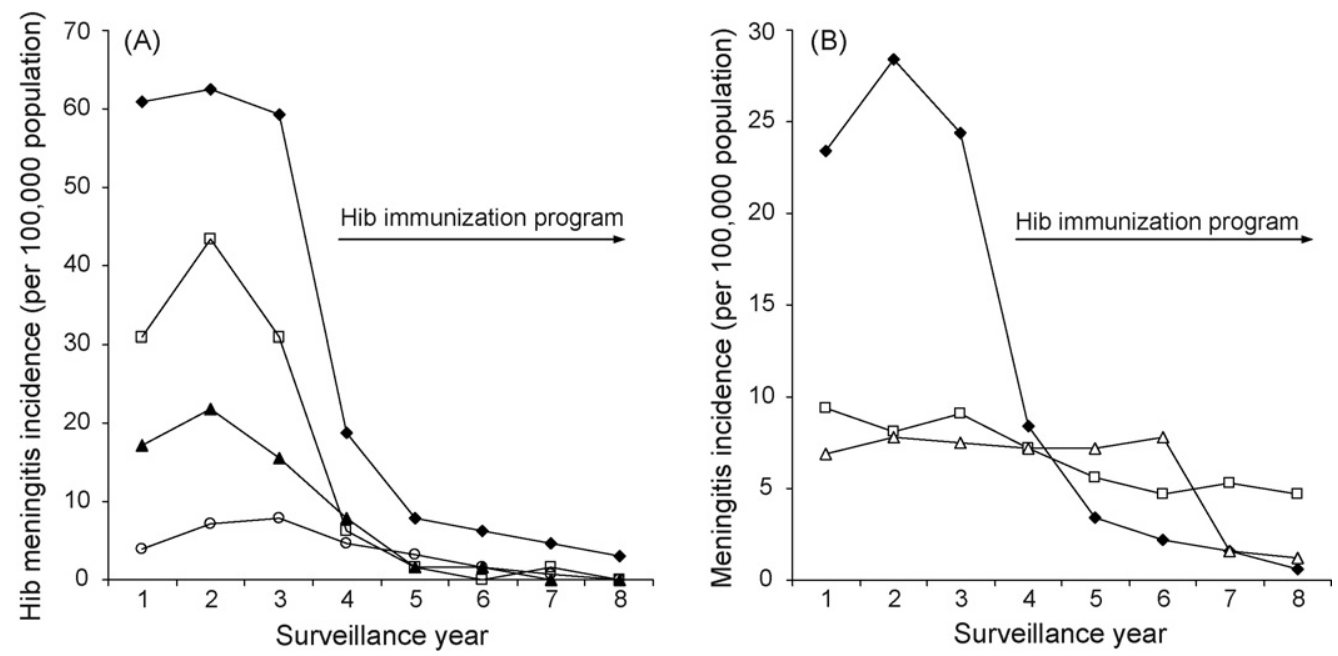

Fig. 2. Incidence of bacterial meningitis in children from Salvador, Brazil for the 8-year period between August 1996 and July 2004. (A) Incidence of Hib meningitis in children $<1$ year (closed lozenge), 1 year (open square), 2 years (closed triangle), and 3 and 4 years (open circle) of age. (B) Incidence of Hib (closed lozenge), Streptococcus pneumoniae (open square) and Neisseria meningitidis (open triangle) meningitis in children $<5$ years of age. The Hib immunization campaign was initiated in August 1999. 
Table 2

Reduction in the Haemophilus influenzae type b (Hib) meningitis incidence after the introduction of Hib immunization in Salvador, Brazil

\begin{tabular}{|c|c|c|c|c|c|}
\hline \multirow[t]{2}{*}{ Age group } & \multicolumn{5}{|l|}{ Surveillance year ${ }^{\mathrm{a}}$} \\
\hline & 4 & 5 & 6 & 7 & 8 \\
\hline Total & $\begin{array}{l}\% \text { Reduction }[95 \% \mathrm{CI}]^{\mathrm{b}} \\
64.6[48.4-75.7]\end{array}$ & $82.3[70.3-89.4]$ & 87.0 [76.4-92.8] & 94.1 [86.1-97.5] & $97.6[91.3-99.4]$ \\
\hline$<5$ years & 66.8 [50.7-77.6] & 86.5 [75.5-92.5] & 91.4 [82.1-95.9] & 93.9 [85.5-97.4] & $97.5[91.0-99.3]$ \\
\hline$<1$ year & 69.2 [44.7-82.9] & 87.2 [69.5-94.6] & 89.7 [73.2-96.1] & 92.3 [77.1-97.4] & $94.9[81.1-98.6]$ \\
\hline 1 year & $82.4[53.5-93.3]$ & $95.6[74.8-99.2]$ & $100.0[\mathrm{NA}]$ & 95.6 [74.8-99.2] & $100.0[\mathrm{NA}]$ \\
\hline 2 years & $57.1[-6.0-82.7]$ & 91.4 [50.5-98.5] & 91.4 [50.5-98.5] & $100.0[\mathrm{NA}]$ & $100.0[\mathrm{NA}]$ \\
\hline $3-4$ years & $25.0[-78.5-68.5]$ & $50.0[-37.9-81.9]$ & $75.0[4.5-93.5]$ & 87.5 [27.1-97.9] & $100.0[\mathrm{NA}]$ \\
\hline 5-9 years & $0.0[-241.4-70.7]$ & $-25.0[-129.5-75.5]$ & $-25.0[-129.5-75.5]$ & 100.0 [NA] & $100.0[\mathrm{NA}]$ \\
\hline
\end{tabular}

Note: NA, not applicable.

a The 5 surveillance years (4-8) corresponds to the period between August 9, 1999 and August 8, 2004.

b Percent reduction and $95 \%$ confidence intervals were calculated by comparing the mean annual incidence of the pre-vaccine surveillance years 1-3 (August 9, 1996 to August 8,1999) with the incidence of each of the post-vaccine surveillance years (4-8).

in this age group was 0.63 cases per 100,000 population in surveillance year 8 (Table 1), which corresponded to a $97.5 \%$ reduction from the mean incidence during the pre-vaccine period (Table 2 and Fig. 2B).

During the 3-year pre-vaccine period, 244 (57.5\%), 85 (20.0\%), $71(16.7 \%)$ and $24(5.7 \%)$ of the 424 bacterial meningitis cases among children $<5$ years of age were caused by $H$. influenzae type b, S. pneumoniae, $N$. meningitidis and other bacterial agents, respectively. In the 4th and 5th years after introduction of the Hib conjugate vaccine (surveillance years 7 and 8), 7 (10.6\%\%), 32 (48.5\%), 9 (13.6\%) and $18(27.3 \%)$ of the 66 cases of bacterial meningitis in children $<5$ years of age were caused by $H$. influenzae type b, S. pneumoniae, $N$. meningitidis and other bacterial agents, respectively. There was a gradual decline $\left(\chi^{2}\right.$ test for trend, $P<0.05)$ in the incidence of pneumococcal meningitis in children $<5$ years of age from 9.38 to 4.69 cases per 100,000 population between the 1 st and 8th surveillance years (Fig. 2B). The incidence of meningococcal meningitis between the 1st and 6th surveillance years ranged from 6.88 to 7.81 cases per 100,000 population and then decreased sharply to 1.56 and 1.25 cases per 100,000 population in the 7th and 8th surveillance years, respectively (Fig. 2B).

\subsection{Hib meningitis in the Hib conjugate vaccine period}

During the post-vaccine period, $55 \mathrm{Hib}$ meningitis cases were eligible to receive a dose of Hib immunization prior to developing meningitis. Of these, $24 \%$ (13 of 55) cases were $<6$ months of age at the time of their hospitalization. Among the 29 (53\% of 55) cases for whom information on immunization status was available, $14(48 \%)$ and $11(38 \%)$ received none or one conjugate vaccine dose, respectively, prior to hospitalization. A single (3.4\% of 29) case of Hib meningitis occurred during the 5-year post-vaccine period among children who completed a three-dose schedule of Hib immunization.

\subsection{Non-type b H. influenzae meningitis}

Surveillance identified 25 cases of $H$. influenzae non-type b meningitis during the 8-year period (Fig. 1). H. influenzae type a meningitis accounted for $19(76 \%)$ cases of which 4 and 15 were identified in the pre and post-vaccine period, respectively (Fig. 1). Geographical clustering was not observed among $H$. influenzae type a cases during the post-vaccine period. The incidence of $H$. influenzae type a meningitis in Metropolitan Salvador increased from 0.01 to 0.14 cases per 100,000 population in the 1st year after introduction of the Hib conjugate vaccine $(P<0.01)$. The largest increase, albeit not significant $(0.00-1.56$ cases per 100,000 population, $P=0.06$ ), occurred in children $<2$ years of age. However, the increase in $H$. influenzae type a meningitis found in the $1 \mathrm{st}$ year after introduction of the Hib conjugate vaccine was not observed in the following 4 surveillance years, during which incidences were between 0.00 and 0.03 cases per 100,000 population.

PFGE analysis showed that the 19 type a strains were distributed among two different typing patterns, A and B, which contained $6(32 \%)$ and $13(68 \%)$ strains, respectively. The strains within each group were closely related ( $\leq 3$ PFGE band difference). PFGE type A and B strains were isolated from cases identified prior to (A, 2 cases; B, 2 cases) and after (A, 4 cases; B, 11 cases) initiation of the Hib immunization campaign. Strains isolated from the $7 \mathrm{H}$. influenzae type a meningitis cases identified in the 2nd to 5 th surveillance years in the post-vaccine period had the same PFGE types (A, 2 strains; B, 5 strains) as strains isolated in the 1st year following introduction of the Hib conjugate vaccine (A, 2 strains; B, 6 strains), the period when a significant increase in $H$. influenzae type a rates was identified.

A history for Hib immunization status was obtained from $39(56 \%)$ of the $70 \mathrm{H}$. influenzae meningitis cases that belonged to a birth cohort that was eligible to receive the conjugate vaccine. Acquisition of $H$. influenzae type a strains was 
significantly associated $(P<0.01)$ with a history of receiving two or more doses of conjugate vaccine prior to developing meningitis. Among $H$. influenzae type a meningitis cases that were identified during the post-vaccine period and belonged to a birth cohort eligible for Hib immunization, $88 \%$ (7 of 8 ) received two or three Hib conjugate vaccine doses prior to hospitalization. In contrast, $16 \%$ (5 of 31) of the H. influenzae non-type a meningitis cases that belonged to a birth cohort eligible for Hib immunization received two or more vaccine doses.

\section{Discussion}

Introduction of Hib conjugate vaccines throughout South and Central America and the Caribbean in the late 1990s has been a major public health achievement. Several studies have reported the significant short-term impact of this initiative [13-21]. The findings of this study from a major Brazilian metropolitan center provides population-based evidence that incorporation of Hib conjugate vaccines in the national childhood immunization program has produced sustained long-term benefit in reducing Hib disease burden, leading towards virtual elimination of this problem for the region.

Five years after initiation of the Hib conjugate vaccine campaign, the Hib meningitis incidence declined by $98 \%$ among children $<5$ years of age in Metropolitan Salvador. We estimate that the use of Hib conjugate vaccines prevented 360 cases of meningitis and 58 deaths in this metropolitan center of 3.5 million inhabitants during the period. Furthermore, conjugate vaccines prevented the severe neurological sequelae encountered in more than $25 \%$ of the survivors of Hib meningitis during the pre-vaccine period. Since active surveillance was performed for meningitis and did not include other invasive diseases associated with $H$. influenzae, the overall benefit of the Hib conjugate vaccine campaign is underestimated. Of note, the Hib meningitis incidence decreased by $82 \%$ within the first 2 years of the campaign.

Overall vaccine coverage among the target population of children $<1$ years of age was $77-82 \%$ during this period. Incomplete coverage includes infants who did not receive any Hib immunization, possibly due to lack of access to health services, and those who received a vaccine dose but did not complete the three dose regimen. Information is not available to evaluate the contribution of each of these groups to the incomplete coverage in the study region. Nevertheless, the ability of the conjugate vaccine to confer herd immunity was presumably a factor in the rapid decline in incidence. In the first year of the campaign, the incidence of Hib meningitis decreased by 57.1 and $25 \%$ among children in the 2 and 3-4 year of age groups, respectively, which were not targeted for immunization. The sharp decline in Hib meningitis incidence may have also been attributable, as observed in other regions [27-29], to the use of a single catch-up dose in infants between 12 and 23 months in the first 2 years of the campaign.
Active surveillance was performed during an 8-year period, in which changes in case ascertainment could have affected the accuracy of the estimate of Hib conjugate vaccine effectiveness. State and local protocols for the referral of suspected meningitis cases to the surveillance hospital did not change during the study period. Review of case notification records did not identify a shift of cases to other hospitals in the region [23]. Antibiotic use prior to hospitalization may have led to reduced culture isolation rates among patients with Hib meningitis and underestimation of the Hib disease burden during the surveillance period. We did not detect a change in the proportion of cases with culture-negative bacterial meningitis in surveys performed at the beginning and end of the surveillance period, which could have arisen due to changes in culture isolation procedures or use of antimicrobial agents prior to hospitalization. A decrease in the incidence for meningococcal and pneumococcal meningitis was observed among children with less than 5 years of age in the absence of any specific preventive intervention against these diseases. However, rates of meningococcal and pneumococcal meningitis did not follow a similar pattern of decrease; neither did they follow the pattern observed for $\mathrm{Hib}$ meningitis. Furthermore, the decrease in meningococcal and pneumococcal meningitis rates appears to be a phenomenon observed throughout Brazil since case notification data has shown a similar, albeit unexplained, trend, at the national level [30,31].

This study found that routine immunization with Hib conjugate vaccines produced a sustained reduction in Hib meningitis disease burden throughout the 5-year period after its introduction. In United Kingdom [32] and Netherlands [33], an increase in Hib invasive diseases incidence was observed 7 and 9 years, respectively, after initiation of Hib immunization. Possible explanations for this increase have been proposed and relate to the decline in immunological memory due to an early and accelerated vaccine schedule $[32,34]$, administration of Hib conjugate vaccine in combination with the acellular pertussis (DTaP) vaccine [32,35], and reduction in natural boosting due to low levels of carriage following vaccine introduction [36]. In Brazil, a three-dose schedule of Hib conjugate vaccine is administered in combination with the whole cell pertussis vaccine (DTwP). There was no increase in Hib meningitis rates identified among birth cohorts who were eligible to receive the conjugate vaccine (Table 1), including the initial cohort of children who were born in the 1st year after introduction of the vaccine and followed for a 5-year period. A single documented case of Hib meningitis was identified among children who completed the three-dose schedule. The study findings do not at present suggest problems with the long-term effectiveness of the conjugate vaccine and immunization schedule used in Brazil and most countries in Latin America. However, the follow-up period has not been sufficient and will require several more years of surveillance to adequately address this issue.

Our prior study in Salvador, Brazil found that a serotype replacement phenomenon occurred after initiation of the 
Hib conjugate vaccine campaign. Identification of this phenomenon was based on the following evidence: (1) a small but significant increase in the $H$. influenzae type a meningitis incidence occurred during the 1-year period following the initiation of the Hib conjugate vaccine campaign; (2) cases of $H$. influenzae type a meningitis were due to two clonal groups that were circulating prior to the introduction of the Hib conjugate vaccine; and (3) prior immunization with two or more vaccine doses was associated with increased risk for $H$. influenzae type a meningitis and therefore appeared to provide a positive selective pressure for type a strains [21]. In this investigation, surveillance performed over an extended 5-year period found a similar association between prior immunization with the Hib conjugate vaccine and the risk for acquiring $H$. influenzae type a meningitis. However, increased rates of $H$. influenzae type a meningitis occurred only in the first year after introduction of the conjugate vaccine and were not observed in the following 4year surveillance period, indicating that serotype replacement was a transient phenomenon. Transmission of $H$. influenzae type a meningitis was due to circulation of two clonal groups throughout the post-vaccine period. The decrease in $H$. influenzae type a meningitis rates after the 1 st year following vaccine introduction presumably relate more to decreased transmission of these clonal groups than changes in the type of conjugate vaccine used or the selective pressure afforded by these vaccines.

Although serotype replacement is a well-recognized phenomenon in association with the use of pneumococcal conjugate vaccines [37], it has not been an important consideration with respect to Hib conjugate vaccines. This may relate to the relatively low virulence of $H$. influenzae non-type $\mathrm{b}$ strains, which frequently produce nasopharyngeal colonization yet infrequently cause invasive disease. $H$. influenzae non-type $b$ invasive disease has been increasingly reported in regions where the Hib conjugate vaccine has been used [38-44]. In addition, non-type b strains have been shown to cause outbreaks and clusters of invasive disease as seen with H. influenzae type a in Gambia [45], Utah [46] and Alaska [47]. In some of these settings, $H$. influenzae type a isolates were found to produce increased amounts of capsule due to amplification of cap loci [48] and/or partial deletion in the IS1016-bexA region within duplicated cap loci [45,46]. Moreover, non-type $b$ isolates have limited genetic diversity, suggesting that increase in invasive disease incidence may be due to the emergence of virulent clones $[38,46,47,49]$. The presence of circulating virulent non-type $b$ strains may therefore be a necessary requirement, in addition to the selection pressure afforded by the use of Hib conjugate vaccines, to produce a serotype replacement phenomenon. The same $H$. influenzae type a clones that led to a serotype replacement phenomenon in Salvador have been isolated in other regions of Brazil [21]. Yet serotype replacement has been a transitory and local phenomenon, not documented outside of Salvador. Furthermore, serotype replacement has not been observed in populations, such as Navajo and White Mountain Apache communities [50], where Hib immunization is routinely used and surveillance has identified the presence of circulating $H$. influenzae type a clones. Together these findings suggest that type a clones may not achieve sufficient levels of transmission to become a significant problem with the use of Hib conjugate vaccines.

In summary, this study found that within a 5-year period, incorporation of Hib conjugate vaccines in childhood immunization program has led to the near elimination of $\mathrm{Hib}$ meningitis in the third largest urban center in Brazil. Overall success of the campaign may be attributed to the high coverage of target populations and the absence of vaccine failure or sustained serotype replacement phenomenon. The benefits afforded by Hib conjugate vaccines, comparable to that which has been achieved in the industrialized world, is likely to be widespread throughout Brazil and other countries in Latin America where the same vaccine regimens are used and similar coverage rates have been attained. The achievements accomplished in Latin America make it ever more imperative to advance on-going initiatives [51] that aim to introduce conjugate vaccines in developing countries where Hib invasive disease continues to cause a major health burden.

\section{Acknowledgements}

This work was supported by grants from the Brazilian National Research Council (300.861/96-6) and National Institute of Health, USA (TW-00919, TW-007303). We thank the clinical, laboratory and administrative staff of Hospital Couto Maia, especially Ana Maria Maia and Neide Oliveira Silva; Steve Copolla for assistance in the data collection and processing; Maviany Mota for assistance with the laboratory analyses; Brendan Flannery for assistance with the statistical analyses; Edgar Lessa Crusoé, Orgalí Marques, Neci Ivo Ramos and Nilda Lúcia Nunes Ivo, from the Secretary of Health for State of Bahia for providing information on the coverage of Hib immunization program and meningitis case notifications; Warren D. Johnson, Jr. and Lee W. Riley for critical advice during study implementation and most of all we thank the study patients and their families.

\section{References}

[1] Progress toward elimination of Haemophilus influenzae type b invasive disease among infants and children, United States, 1998-2000. MMWR Morb Mortal Wkly Rep 2002; 51(11):234-7.

[2] Peltola H. Worldwide Haemophilus influenzae type b disease at the beginning of the 21st century: global analysis of the disease burden 25 years after the use of the polysaccharide vaccine and a decade after the advent of conjugates. Clin Microbiol Rev 2000;13(2):302-17.

[3] Schuchat A, Robinson K, Wenger JD, Harrison LH, Farley M, Reingold AL, et al. Bacterial meningitis in the United States in 1995. Active Surveillance Team. N Engl J Med 1997;337(14):970-6.

[4] Santosham M, Wolff M, Reid R, Hohenboken M, Bateman M, Goepp $\mathrm{J}$, et al. The efficacy in Navajo infants of a conjugate vaccine consisting of Haemophilus influenzae type b polysaccharide and Neis- 
seria meningitidis outer-membrane protein complex. N Engl J Med 1991;324(25):1767-72.

[5] Eskola J, Kayhty H, Takala AK, Peltola H, Ronnberg PR, Kela E, et al. A randomized, prospective field trial of a conjugate vaccine in the protection of infants and young children against invasive Haemophilus influenzae type b disease. N Engl J Med 1990;323(20):1381-7.

[6] Barbour ML, Mayon-White RT, Coles C, Crook DW, Moxon ER. The impact of conjugate vaccine on carriage of Haemophilus influenzae type b. J Infect Dis 1995;171(1):93-8.

[7] Forleo-Neto E, de Oliveira CF, Maluf EM, Bataglin C, Araujo JM, Kunz Jr LF, et al. Decreased point prevalence of Haemophilus influenzae type $\mathrm{b}(\mathrm{Hib})$ oropharyngeal colonization by mass immunization of Brazilian children less than 5 years old with hib polyribosylribitol phosphate polysaccharide-tetanus toxoid conjugate vaccine in combination with diphtheria-tetanus toxoids-pertussis vaccine. J Infect Dis 1999;180(4):1153-8.

[8] Adegbola RA, Secka O, Lahai G, Lloyd-Evans N, Njie A, Usen S, et al. Elimination of Haemophilus influenzae type $\mathrm{b}(\mathrm{Hib})$ disease from The Gambia after the introduction of routine immunisation with a Hib conjugate vaccine: a prospective study. Lancet 2005;366(9480):144-50.

[9] Moulton LH, Chung S, Croll J, Reid R, Weatherholtz RC, Santosham M. Estimation of the indirect effect of Haemophilus influenzae type b conjugate vaccine in an American Indian population. Int J Epidemiol 2000;29(4):753-6.

[10] World Health Organization. WHO vaccine-preventable diseases: monitoring system, 2004 global summary. Website: http://www.who. int/immunization_delivery/systems_policy/GlobalSummary2004.pdf [last accessed November 06, 2006].

[11] World Health Organization. Estimates of disease burden and costeffectiveness. Website: http://www.who.int/immunization_monitoring/ burden/estimates_burden/en/[last accessed November 06, 2006].

[12] World Health Organization. WHO UNICEF review of national immunization coverage, 1980-2004. Website: http://www.who.int/ immunization_monitoring/en/globalsummary/wucoveragecountrylist. cfm\#Latin\%20America\%20and\%20the\%20Caribean [last accessed November 06, 2006].

[13] Dickinson FO, Perez AE, Galindo MA, Quintana I. Impact of vaccination against Haemophilus influenzae type b in Cuba. Rev Panam Salud Publica 2001;10(3):169-73.

[14] Kmetzsch CI, Schermann MT, Santana JC, Estima CL, Faraco FJ, Silva $\mathrm{CM}$, et al. Occurrence of Haemophylus influenzae B meningitis after the implementation of a mass vaccination program. J Pediatr (Rio J) 2003;79(6):530-6.

[15] Lagos R, Horwitz I, Toro J, San Martin O, Abrego P, Bustamante C, et al. Large scale, postlicensure, selective vaccination of Chilean infants with PRP-T conjugate vaccine: practicality and effectiveness in preventing invasive Haemophilus influenzae type b infections. Pediatr Infect Dis J 1996;15(3):216-22.

[16] Ruocco G, Curto S, Savio M, Laurani H, Frocht R. Vaccination against Haemophilus influenzae type b in Uruguay: experience and impact. Rev Panam Salud Publica 1999;5(3):197-9.

[17] Simoes LL, Andrade AL, Laval CA, Oliveira RM, Silva SA, Martelli $\mathrm{CM}$, et al. Impact of Haemophilus influenzae b (Hib) vaccination on meningitis in Central Brazil. Rev Saude Publica 2004;38(5):664-70.

[18] Takemura NS, Andrade SM. Haemophilus influenzae type b meningitis in the state of Parana. Brazil J Pediatr (Rio J) 2001;77(5): 387-92.

[19] Freitas HS, Merchan-Hamann E. Impact of anti-Hib conjugate vaccine on the incidence of Haemophilus influenzae meningitis in Brazil's Federal District: results of a three-year follow-up. Rev Panam Salud Publica 2006;19(1):33-7.

[20] de Andrade AL, de Andrade JG, Martelli CM, e Silva SA, de Oliveira $\mathrm{RM}$, Costa MS, et al. Effectiveness of Haemophilus influenzae b conjugate vaccine on childhood pneumonia: a case-control study in Brazil. Int J Epidemiol 2004;33(1):173-81.

[21] Ribeiro GS, Reis JN, Cordeiro SM, Lima JB, Gouveia EL, Petersen M, et al. Prevention of Haemophilus influenzae type b (Hib) meningitis and emergence of serotype replacement with type a strains after introduction of Hib immunization in Brazil. J Infect Dis 2003;187(1):109-16.

[22] Instituto Brasileiro de Geografia e Estatística. Censo Demográfico 2000 — Resultados do universo. Rio de Janeiro: Instituto Brasileiro de Geografia e Estatística; 2002.

[23] Case notification records. Salvador, Brazil: Epidemiological Surveillance Division, Secretary of Health for the State of Bahia; 2005.

[24] Immunization records. Salvador, Brazil: Epidemiological Surveillance Division, Secretary of Health for the State of Bahia; 2005.

[25] Falla TJ, Crook DW, Brophy LN, Maskell D, Kroll JS, Moxon ER. PCR for capsular typing of Haemophilus influenzae. J Clin Microbiol 1994;32(10):2382-6.

[26] Tenover FC, Arbeit RD, Goering RV, Mickelsen PA, Murray BE, Persing DH, et al. Interpreting chromosomal DNA restriction patterns produced by pulsed-field gel electrophoresis: criteria for bacterial strain typing. J Clin Microbiol 1995;33(9):2233-9.

[27] Hviid A, Melbye M. Impact of routine vaccination with a conjugate Haemophilus influenzae type b vaccine. Vaccine 2004;22(3-4):378-82.

[28] Rushdy A, Ramsay M, Heath PT, Azzopardi HJ, Slack MP. Infant Hib vaccination and herd immunity. J Pediatr 1999;134(2):253-4.

[29] van Alphen L, Spanjaard L, van der Ende A, Schuurman I, Dankert J. Effect of nationwide vaccination of 3-month-old infants in The Netherlands with conjugate Haemophilus influenzae type b vaccine: high efficacy and lack of herd immunity. J Pediatr 1997;131(6):869-73.

[30] Centro de Vigilância Epidemiológica do Estado de São Paulo. Meningites/Doença Meningocócica - Dados Estatísticos. Website: http://www.cve.saude.sp.gov.br/htm/resp/meni_dados.html [last accessed November 06, 2006].

[31] Datasus, Ministério da Saúde do Brasil. Informações de Saúde: Epidemiológicas e Morbidade. Website: http://w3.datasus.gov.br/ datasus/datasus.php?area=359A1B624C4D0E0F359G9H0I1Jd4L24 M0N\&VInclude=./site/infsaude.php\&VObj=http://tabnet.datasus.gov. $\mathrm{br} / \mathrm{cgi} / \mathrm{deftohtm}$. exe? $\mathrm{sih} / \mathrm{cnv} / \mathrm{mr}$ [last accessed November 06, 2006].

[32] Ramsay ME, McVernon J, Andrews NJ, Heath PT, Slack MP. Estimating Haemophilus influenzae type b vaccine effectiveness in England and Wales by use of the screening method. J Infect Dis 2003;188(4):481-5.

[33] Rijkers GT, Vermeer-de Bondt PE, Spanjaard L, Breukels MA, Sanders EA. Return of Haemophilus influenzae type b infections. Lancet 2003;361(9368):1563-4.

[34] Trotter CL, McVernon J, Andrews NJ, Burrage M, Ramsay ME. Antibody to Haemophilus influenzae type b after routine and catch-up vaccination. Lancet 2003;361(9368):1523-4.

[35] McVernon J, Andrews N, Slack MP, Ramsay ME. Risk of vaccine failure after Haemophilus influenzae type b (Hib) combination vaccines with acellular pertussis. Lancet 2003;361(9368):1521-3.

[36] McVernon J, Mitchison NA, Moxon ER. T helper cells and efficacy of Haemophilus influenzae type b conjugate vaccination. Lancet Infect Dis 2004;4(1):40-3.

[37] Direct and indirect effects of routine vaccination of children with 7 -valent pneumococcal conjugate vaccine on incidence of invasive pneumococcal disease, United States, 1998-2003. MMWR Morb Mortal Wkly Rep 2005; 54(36):893-7.

[38] Tsang RS, Mubareka S, Sill ML, Wylie J, Skinner S, Law DK. Invasive Haemophilus influenzae in Manitoba, Canada, in the postvaccination era. J Clin Microbiol 2006;44(4):1530-5.

[39] Bajanca P, Canica M. Emergence of nonencapsulated and encapsulated non-b-type invasive Haemophilus influenzae isolates in Portugal (1989-2001). J Clin Microbiol 2004;42(2):807-10.

[40] de Almeida AE, de Filippis I, Abreu AO, Ferreira DG, Gemal AL, Marzochi KB. Occurrence of Haemophilus influenzae strains in three Brazilian states since the introduction of a conjugate Haemophilus influenzae type b vaccine. Braz J Med Biol Res 2005;38(5): 777-81.

[41] Heath PT, Booy R, Azzopardi HJ, Slack MP, Fogarty J, Moloney AC, et al. Non-type b Haemophilus influenzae disease: clinical and epidemiologic characteristics in the Haemophilus influenzae type b vaccine era. Pediatr Infect Dis J 2001;20(3):300-5. 
[42] Perdue DG, Bulkow LR, Gellin BG, Davidson M, Petersen KM, Singleton RJ, et al. Invasive Haemophilus influenzae disease in Alaskan residents aged 10 years and older before and after infant vaccination programs. JAMA 2000;283(23):3089-94.

[43] Urwin G, Krohn JA, Deaver-Robinson K, Wenger JD, Farley MM. Invasive disease due to Haemophilus influenzae serotype f: clinical and epidemiologic characteristics in the $H$. influenzae serotype b vaccine era. The Haemophilus influenzae Study Group. Clin Infect Dis 1996;22(6):1069-76.

[44] Waggoner-Fountain LA, Hendley JO, Cody EJ, Perriello VA, Donowitz LG. The emergence of Haemophilus influenzae types e and $\mathrm{f}$ as significant pathogens. Clin Infect Dis 1995;21(5):1322-4.

[45] Kroll JS, Moxon ER, Loynds BM. Natural genetic transfer of a putative virulence-enhancing mutation to Haemophilus influenzae type a. J Infect Dis 1994;169(3):676-9.

[46] Adderson EE, Byington CL, Spencer L, Kimball A, Hindiyeh M, Carroll $\mathrm{K}$, et al. Invasive serotype a Haemophilus influenzae infections with a virulence genotype resembling Haemophilus influenzae type b: emerging pathogen in the vaccine era? Pediatrics 2001;108(1): E18.

[47] Hammitt LL, Block S, Hennessy TW, Debyle C, Peters H, Parkinson A, et al. Outbreak of invasive Haemophilus influenzae serotype a disease. Pediatr Infect Dis J 2005;24(5):453-6.

[48] Ogilvie C, Omikunle A, Wang Y, St Geme 3rd IJ, Rodriguez CA, Adderson EE. Capsulation loci of non-serotype b encapsulated Haemophilus influenzae. J Infect Dis 2001;184(2):144-9.

[49] Omikunle A, Takahashi S, Ogilvie CL, Wang Y, Rodriguez CA, St Geme 3rd JW, et al. Limited genetic diversity of recent invasive isolates of non-serotype b encapsulated Haemophilus influenzae. J Clin Microbiol 2002;40(4):1264-70.

[50] Millar EV, O’Brien KL, Watt JP, Lingappa J, Pallipamu R, Rosenstein $\mathrm{N}$, et al. Epidemiology of invasive Haemophilus influenzae type A disease among Navajo and White Mountain Apache children, 1988-2003. Clin Infect Dis 2005;40(6):823-30.

[51] Mitchell V, Walker D, Zuber P, Lydon P, Ahun M. Evidenced-based decision making about Hib vaccination. Lancet 2005;365(9463):936-7. 